\title{
VARIANCE OF THE EXPONENTS OF ORBIFOLD LANDAU-GINZBURG MODELS
}

\author{
Wolfgang Ebeling And Atsushi TAKahashi
}

\begin{abstract}
We prove a formula for the variance of the set of exponents of a nondegenerate weighted homogeneous polynomial with an action of a diagonal subgroup of $\mathrm{SL}_{n}(\mathbb{C})$.
\end{abstract}

\section{Introduction}

Let $X$ be a smooth compact Kähler manifold of dimension $n$. The Hodge numbers $h^{p, q}(X):=\operatorname{dim}_{\mathbb{C}} H^{q}\left(X, \Omega_{X}^{p}\right), p, q \in \mathbb{Z}$, are some of the most important numerical invariants of $X$. They satisfy

$$
h^{p, q}(X)=h^{q, p}(X), \quad p, q \in \mathbb{Z}
$$

and the Serre duality

$$
h^{p, q}(X)=h^{n-p, n-q}(X), \quad p, q \in \mathbb{Z} .
$$

The Euler number $\chi(X)$ can also be written in terms of the Hodge numbers as

$$
\chi(X)=\sum_{p, q \in \mathbb{Z}}(-1)^{p+q} h^{p, q}(X) .
$$

One can easily calculate the expectation value of the distribution $\left\{q \in \mathbb{Z} \mid h^{p, q}(X) \neq\right.$ $0\}$, which is given by the formula

$$
\sum_{p, q \in \mathbb{Z}}(-1)^{p+q} q \cdot h^{p, q}(X)=\frac{1}{2} n \cdot \chi(X) .
$$

Equivalently, this can be rewritten as

$$
\sum_{p, q \in \mathbb{Z}}(-1)^{p+q}\left(q-\frac{n}{2}\right) h^{p, q}(X)=0 .
$$

This means nothing else but that the mean of the distribution $\left\{q \in \mathbb{Z} \mid h^{p, q}(X) \neq 0\right\}$ is $n / 2$. It is then natural to ask what is the variance of this distribution. A formula for this variance was given by Libgober and Wood [9] and Borisov [2]:

Theorem 1 (Libgober-Wood, Borisov). One has

$$
\sum_{p, q \in \mathbb{Z}}(-1)^{p+q}\left(q-\frac{n}{2}\right)^{2} h^{p, q}(X)=\frac{1}{12} n \cdot \chi(X)+\frac{1}{6} \int_{X} c_{1}(X) \cup c_{n-1}(X),
$$

where $c_{i}(X)$ denotes the ith Chern class of $X$.

Received by the editors June 22, 2012.

2010 Mathematics Subject Classification. 32S25, 32S35, 14L30. 
If the first Chern class, $c_{1}(X)$ is numerically zero, then the above formula becomes

$$
\sum_{p, q \in \mathbb{Z}}(-1)^{p+q}\left(q-\frac{n}{2}\right)^{2} h^{p, q}(X)=\frac{1}{12} n \cdot \chi(X) .
$$

Similar phenomena were discovered in singularity theory. Let us consider a polynomial $f\left(x_{1}, \ldots, x_{n}\right)$ with an isolated singularity at the origin. There, the analogue of the set $\left\{q \in \mathbb{Z} \mid h^{p, q}(X) \neq 0\right\}$ above will be the set of the exponents of $f\left(x_{1}, \ldots, x_{n}\right)$, which is a set of rational numbers and is also one of the most important numerical invariants defined by the mixed Hodge structure associated to $f\left(x_{1}, \ldots, x_{n}\right)$. Let us give two important examples.

First, suppose that $f\left(x_{1}, \ldots, x_{n}\right)$ is a non-degenerate weighted homogeneous polynomial, namely, a polynomial with an isolated singularity at the origin with the property that there are positive rational numbers $w_{i}, i=1, \ldots, n$, such that

$$
f\left(\lambda^{w_{1}} x_{1}, \ldots, \lambda^{w_{n}} x_{n}\right)=\lambda f\left(x_{1}, \ldots, x_{n}\right), \quad \lambda \in \mathbb{C} \backslash\{0\} .
$$

We have the following properties of the exponents of $f$ :

Theorem 2 (cf. [10]). Let $q_{1} \leq q_{2} \leq \cdots \leq q_{\mu}$ be the exponents of $f$, where $\mu$ is the Milnor number of $f$ defined by

$$
\mu:=\operatorname{dim}_{\mathbb{C}} \mathbb{C}\left[x_{1}, \ldots, x_{n}\right] /\left(\frac{\partial f}{\partial x_{1}}, \ldots, \frac{\partial f}{\partial x_{n}}\right) .
$$

Then one has

$$
\mu=(-1)^{n} \prod_{i=1}^{n}\left(1-\frac{1}{w_{i}}\right)
$$

and

$$
\sum_{i=1}^{\mu} y^{q_{i}-\frac{n}{2}}=(-1)^{n} \prod_{i=1}^{n} \frac{y^{\frac{1}{2}}-y^{w_{i}-\frac{1}{2}}}{1-y^{w_{i}}} .
$$

In particular, one has a duality of exponents $q_{i}+q_{\mu-i+1}=n, i=1, \ldots, \mu$, and hence

$$
\sum_{i=1}^{\mu} q_{i}=\frac{1}{2} n \cdot \mu \text {. }
$$

The following formula was proven by Hertling [6] in the context of Frobenius manifolds and an elementary proof was given by Dimca [3].

Theorem 3 (Hertling, Dimca). Let $q_{1} \leq q_{2} \leq \cdots \leq q_{\mu}$ be the exponents of $f$. One has

$$
\sum_{i=1}^{\mu}\left(q_{i}-\frac{n}{2}\right)^{2}=\frac{1}{12} \hat{c} \cdot \mu, \quad \hat{c}:=n-2 \sum_{i=1}^{n} w_{i} .
$$

Next, consider the polynomial $f\left(x_{1}, x_{2}, x_{3}\right):=x_{1}^{\alpha_{1}}+x_{2}^{\alpha_{2}}+x_{3}^{\alpha_{3}}-x_{1} x_{2} x_{3}$ such that $1 / \alpha_{1}+1 / \alpha_{2}+1 / \alpha_{3}<1$. We have the following properties of the exponents of $f$ : 
Theorem 4 (cf. [1]). The set of exponents $\left\{q_{i}\right\}$ of $f$ is given by

$$
\begin{aligned}
\left\{1, \frac{1}{\alpha_{1}}+1, \frac{2}{\alpha_{1}}+1, \ldots, \frac{\alpha_{1}-1}{\alpha_{1}}+1, \frac{1}{\alpha_{2}}+1, \frac{2}{\alpha_{2}}+1,\right. \\
\left.\quad \ldots, \frac{\alpha_{2}-1}{\alpha_{2}}+1, \frac{1}{\alpha_{3}}+1, \frac{2}{\alpha_{3}}+1, \ldots, \frac{\alpha_{3}-1}{\alpha_{3}}+1,2\right\} .
\end{aligned}
$$

In particular, one has

$$
\sum_{i=1}^{\mu}\left(q_{i}-\frac{3}{2}\right)^{2}=\frac{1}{12} \mu+\frac{1}{6} \chi, \quad \chi:=2+\sum_{i=1}^{3}\left(\frac{1}{\alpha_{i}}-1\right) .
$$

The purpose of this paper is to generalize these results to pairs $(f, G)$, where $G \subset \mathrm{SL}_{n}(\mathbb{C})$ is a finite abelian subgroup leaving $f$ invariant. If $f$ is weighted homogeneous, such a pair is also called an orbifold Landau-Ginzburg model because $f$ is the potential of such a model. Our main theorem in this paper is Theorem 19. The generalization of Theorem 4 is given as Theorem 21. The similarity between smooth compact Kähler manifolds and isolated hypersurface singularities with a group action is not an accident but a matter of course. Mirror symmetry predicts a correspondence between Landau-Ginzburg models and (non-commutative) Calabi-Yau orbifolds. For example, a mirror partner of a weighted homogeneous polynomial with a group action is a fractional Calabi-Yau manifold of dimension $\hat{c}$, which has lead us to the statement of Theorem 19.

\section{Basic properties of E-functions}

Let $G$ be a finite abelian subgroup of $\mathrm{SL}_{n}(\mathbb{C})$ acting diagonally on $\mathbb{C}^{n}$. For $g \in G$, we denote by Fix $g:=\left\{x \in \mathbb{C}^{n} \mid g \cdot x=x\right\}$ the fixed locus of $g$ and by $n_{g}:=\operatorname{dim}$ Fix $g$ its dimension.

We first introduce the notion of the age of an element of a finite group as follows:

Definition ([8]). Let $g \in G$ be an element and $r$ be the order of $g$. Then $g$ has a unique expression of the following form:

$$
g=\operatorname{diag}\left(\mathbf{e}\left[a_{1} / r\right], \ldots, \mathbf{e}\left[a_{n} / r\right]\right) \quad \text { with } 0 \leq a_{i}<r,
$$

where $\mathbf{e}[-]=e^{2 \pi \sqrt{-1} \cdot-}$. Such an element $g$ is often simply denoted by

$$
g=\frac{1}{r}\left(a_{1}, \ldots, a_{n}\right) .
$$

The age of $g$ is defined as

$$
\operatorname{age}(g):=\frac{1}{r} \sum_{i=1}^{n} a_{i} .
$$

Since we assume that $G \subset \mathrm{SL}_{n}(\mathbb{C})$, the number age $(g)$ is a non-negative integer for all $g \in G$.

Definition. An element $g \in G$ of age 1 with Fix $g=\{0\}$ is called a junior element. The number of junior elements is denoted by $j_{G}$.

Let $f=f\left(x_{1}, \ldots, x_{n}\right)$ be a polynomial with an isolated singularity at the origin, which is invariant under the natural action of $G$. For $g \in G$, set $f^{g}:=\left.f\right|_{\text {Fix } g}$. 
Proposition 5. The function $f^{g}$ has an isolated singularity at the origin.

Proof. Since $G$ acts diagonally on $\mathbb{C}^{n}$, we may assume that Fix $g=\left\{x_{n_{g}+1}=\cdots=\right.$ $\left.x_{n}=0\right\}$ by a suitable renumbering of indices. Since $f$ is invariant under $G, g \cdot x_{i} \neq x_{i}$ for $i=n_{g}+1, \ldots, n$ and $\frac{\partial f}{\partial x_{n_{g}+1}}, \ldots, \frac{\partial f}{\partial x_{n}}$ form a regular sequence, we have

$$
\left(\frac{\partial f}{\partial x_{n_{g}+1}}, \ldots, \frac{\partial f}{\partial x_{n}}\right) \subset\left(x_{n_{g}+1}, \ldots, x_{n}\right) .
$$

Therefore, we have

$$
\begin{array}{rl}
\operatorname{dim}_{\mathbb{C}} & \mathbb{C}\left\{x_{1}, \ldots, x_{n_{g}}\right\} /\left(\frac{\partial f^{g}}{\partial x_{1}}, \ldots, \frac{\partial f^{g}}{\partial x_{n_{g}}}\right) \\
& =\operatorname{dim}_{\mathbb{C}} \mathbb{C}\left\{x_{1}, \ldots, x_{n}\right\} /\left(\frac{\partial f}{\partial x_{1}}, \ldots, \frac{\partial f}{\partial x_{n_{g}}}, x_{n_{g}+1}, \ldots, x_{n}\right) \\
& \leq \operatorname{dim}_{\mathbb{C}} \mathbb{C}\left\{x_{1}, \ldots, x_{n}\right\} /\left(\frac{\partial f}{\partial x_{1}}, \ldots, \frac{\partial f}{\partial x_{n}}\right)<\infty
\end{array}
$$

We shall associate to $f$ the following bi-graded vector space:

Definition. Let $H^{n-1}\left(Y_{\infty}, \mathbb{C}\right)$ be the vanishing cohomology of $f$ on which Steenbrink constructed a canonical mixed Hodge structure in [10]. Denote by $F^{\bullet}$ the Hodge filtration on $H^{n-1}\left(Y_{\infty}, \mathbb{C}\right)$.

Define the bi-graded vector space $\mathcal{H}_{f}:=\bigoplus_{p, q \in \mathbb{Q}} \mathcal{H}_{f}^{p, q}$ as

(i) If $p+q \neq n$, then $\mathcal{H}_{f}^{p, q}:=0$.

(ii) If $p+q=n$ and $p \in \mathbb{Z}$, then

$$
\mathcal{H}_{f}^{p, q}:=\mathrm{Gr}_{F}^{p} \bullet H^{n-1}\left(Y_{\infty}, \mathbb{C}\right)_{1} .
$$

(iii) If $p+q=n$ and $p \notin \mathbb{Z}$, then

$$
\mathcal{H}_{f}^{p, q}:=\operatorname{Gr}_{F}^{[p]} H^{n-1}\left(Y_{\infty}, \mathbb{C}\right)_{e^{2 \pi \sqrt{-1} p}},
$$

where $[p]$ is the largest integer less than $p$.

We shall use the fact that $\mathcal{H}_{f^{g}}$ admits a natural $G$-action by restricting the $G$-action on $\mathbb{C}^{n}$ to Fix $g$ (which is well-defined since $G$ acts diagonally on $\mathbb{C}^{n}$ ).

To the pair $(f, G)$ we can associate the following bi-graded vector space:

Definition. Define the bi-graded $\mathbb{C}$-vector space $\mathcal{H}_{f, G}$ as

$$
\mathcal{H}_{f, G}:=\bigoplus_{g \in G}\left(\mathcal{H}_{f^{g}}\right)^{G}(-\operatorname{age}(g),-\operatorname{age}(g)),
$$

where $\left(\mathcal{H}_{f^{g}}\right)^{G}$ denotes the $G$-invariant subspace of $\mathcal{H}_{f^{g}}$.

Since the bi-graded vector space $\mathcal{H}_{f, G}$ is the analog of $\bigoplus_{p, q \in \mathbb{Z}} H^{q}\left(X, \Omega_{X}^{p}\right)$ for a smooth compact Kähler manifold $X$, we introduce the following notion:

Definition. The Hodge numbers for the pair $(f, G)$ are

$$
h^{p, q}(f, G):=\operatorname{dim}_{\mathbb{C}} \mathcal{H}_{f, G}^{p, q}, \quad p, q \in \mathbb{Q} .
$$


Definition. The rational number $q$ with $\mathcal{H}_{f, G}^{p, q} \neq 0$ is called an exponent of the pair $(f, G)$. The set of exponents of the pair $(f, G)$ is the multi-set of exponents

$$
\left\{q * h^{p, q}(f, G) \mid p, q \in \mathbb{Q}, h^{p, q}(f, G) \neq 0\right\},
$$

where by $u * v$ we denote $v$ copies of the rational number $u$.

Note that $p+q \in \mathbb{Z}$ for the rational number $q$ with $h^{p, q}(f, G) \neq 0$ since $G \subset \mathrm{SL}_{n}(\mathbb{C})$.

Definition. The E-function for the pair $(f, G)$ is

$$
E(f, G)(t, \bar{t}):=\sum_{p, q \in \mathbb{Q}}(-1)^{(p-n)+q} h^{p, q}(f, G) \cdot t^{p-\frac{n}{2}} \bar{t}^{q-\frac{n}{2}} .
$$

Definition. The Milnor number for the pair $(f, G)$ is

$$
\mu_{(f, G)}:=E(f, G)(1,1)=\sum_{p, q \in \mathbb{Q}}(-1)^{(p-n)+q} h^{p, q}(f, G) .
$$

Theorem 6. Assume that $f$ is a non-degenerate weighted homogeneous polynomial. Write $g \in G$ in the form $\left(\lambda_{1}(g), \ldots, \lambda_{n}(g)\right)$ where $\lambda_{i}(g)=\mathbf{e}\left[a_{i} w_{i}\right]$. The E-function for the pair $(f, G)$ is given by the following formula:

$$
\begin{gathered}
E(f, G)(t, \bar{t})=\sum_{g \in G} E_{g}(f, G)(t, \bar{t}), \\
E_{g}(f, G)(t, \bar{t}):=(-1)^{n}\left(\prod_{a_{i} w_{i} \notin \mathbb{Z}}(t \bar{t})^{w_{i} a_{i}-\left[w_{i} a_{i}\right]-\frac{1}{2}}\right) \\
\cdot \frac{1}{|G|} \sum_{h \in G} \prod_{a_{i} w_{i} \in \mathbb{Z}} \frac{\left(\frac{\bar{t}}{t}\right)^{\frac{1}{2}}-\lambda_{i}(h)\left(\frac{\bar{t}}{t}\right)^{w_{i}-\frac{1}{2}}}{1-\lambda_{i}(h)\left(\frac{\bar{t}}{t}\right)^{w_{i}}} .
\end{gathered}
$$

Here $[a]$ for $a \in \mathbb{Q}$ denotes the largest integer less than or equal to a.

Proof. Theorem 2 enables us to obtain $E_{g}(f, G)(t, \bar{t})$. In particular, the term

$$
\frac{1}{|G|} \sum_{h \in G}(-1)^{n_{g}} \prod_{a_{i} w_{i} \in \mathbb{Z}} \frac{\left(\frac{\bar{t}}{t}\right)^{\frac{1}{2}}-\lambda_{i}(h)\left(\frac{\bar{t}}{t}\right)^{w_{i}-\frac{1}{2}}}{1-\lambda_{i}(h)\left(\frac{\bar{t}}{t}\right)^{w_{i}}}
$$

calculates the $G$-invariant part of $E\left(f^{g},\{1\}\right)(t, \bar{t})$ and the term

$$
(-1)^{n-n_{g}} \prod_{w_{i} a_{i} \notin \mathbb{Z}}(t \bar{t})^{w_{i} a_{i}-\left[w_{i} a_{i}\right]-\frac{1}{2}}
$$

gives the contribution from the age shift $(-\operatorname{age}(g),-\operatorname{age}(g))$.

We have the following properties of the Hodge numbers $h^{p, q}(f, G)$.

Corollary 7. Assume that $f$ is a non-degenerate weighted homogeneous polynomial. We have

$$
h^{p, q}(f, G)=h^{q, p}(f, G), \quad p, q \in \mathbb{Q} .
$$

In other words, we have

$$
E(f, G)(t, \bar{t})=E(f, G)(\bar{t}, t)
$$


Proof. This is shown by an elementary direct calculation.

Corollary 8. Assume that $f$ is a non-degenerate weighted homogeneous polynomial. The Hodge numbers satisfy the "Serre duality"

$$
h^{p, q}(f, G)=h^{n-p, n-q}(f, G), \quad p, q \in \mathbb{Q} .
$$

In other words, we have

$$
E(f, G)(t, \bar{t})=E(f, G)\left(t^{-1}, \bar{t}^{-1}\right) .
$$

Proof. By using the formula

$$
w_{i}\left(-a_{i}\right)-\left[w_{i}\left(-a_{i}\right)\right]-\frac{1}{2}=-w_{i} a_{i}+\left[w_{i} a_{i}\right]+\frac{1}{2},
$$

an easy calculation yields the formula.

Corollary 9. Assume that $f$ is a non-degenerate weighted homogeneous polynomial. The mean of the set of exponents of $(f, G)$ is $n / 2$. Namely, we have

$$
\sum_{p, q \in \mathbb{Q}}(-1)^{(p-n)+q}\left(q-\frac{n}{2}\right) h^{p, q}(f, G)=0 .
$$

Proof. This is obvious from the previous corollary.

Definition. Define the variance of the set of exponents of $(f, G)$ by

$$
\operatorname{Var}_{(f, G)}:=\sum_{p, q \in \mathbb{Q}}(-1)^{(p-n)+q}\left(q-\frac{n}{2}\right)^{2} h^{p, q}(f, G) .
$$

In order to state our formula for the variance, we introduce the following notion of dimension for a polynomial $f$ with an isolated singularity at the origin.

Definition. The non-negative rational number $\hat{c}$ defined as the difference of the maximal exponent of the pair $(f,\{1\})$ and the minimal exponent of the pair $(f,\{1\})$ is called the dimension of $f$.

Proposition 10. Assume that $f$ is a non-degenerate weighted homogeneous polynomial. The dimension $\hat{c}$ of $f$ is given by

$$
\hat{c}:=n-2 \sum_{i=1}^{n} w_{i}
$$

Proof. It easily follows from Theorem 2 that the maximal exponent and the minimal exponent are given by $n-\sum_{i=1}^{n} w_{i}$ and $\sum_{i=1}^{n} w_{i}$, respectively.

It is natural from the mirror symmetry point of view to expect that the variance of the set of exponents of $(f, G)$ should be given by

$$
\operatorname{Var}_{(f, G)}=\frac{1}{12} \hat{c} \cdot \mu_{(f, G)} .
$$

This will be proved in the next section. 


\section{Variance of the exponents}

Definition. The $\chi_{y}$-genus for the pair $(f, G)$ is

$$
\chi(f, G)(y):=E(f, G)(1, y) .
$$

We have

$$
\chi(f, G)(y)=(-1)^{n} \sum_{g \in G}\left(y^{\operatorname{age}(g)-\frac{n-n_{g}}{2}} \cdot \frac{1}{|G|} \sum_{h \in G} \prod_{\lambda_{i}(g)=1} \frac{y^{\frac{1}{2}}-\lambda_{i}(h) y^{w_{i}-\frac{1}{2}}}{1-\lambda_{i}(h) y^{w_{i}}}\right) .
$$

One has

$$
\begin{aligned}
\mu_{(f, G)} & =\lim _{y \rightarrow 1} \chi(f, G)(y), \\
\operatorname{Var}_{(f, G)} & =\lim _{y \rightarrow 1} \frac{d}{d y}\left(y \frac{d}{d y} \chi(f, G)(y)\right) .
\end{aligned}
$$

Proposition 11. Let

$$
p_{i}(y):=\frac{y^{\frac{1}{2}}-\lambda_{i}(h) y^{w_{i}-\frac{1}{2}}}{1-\lambda_{i}(h) y^{w_{i}}} .
$$

(i) For $\lambda_{i}(h)=1$ one has

$$
\lim _{y \rightarrow 1} p_{i}(y)=1-\frac{1}{w_{i}}, \quad \lim _{y \rightarrow 1} \frac{\frac{d}{d y} p_{i}(y)}{p_{i}(y)}=0, \quad \lim _{y \rightarrow 1} \frac{d}{d y}\left(y \frac{\frac{d}{d y} p_{i}(y)}{p_{i}(y)}\right)=\frac{1-2 w_{i}}{12} .
$$

(ii) For $\lambda_{i}(h) \neq 1$ one has

$$
\begin{aligned}
& \lim _{y \rightarrow 1} p_{i}(y)=1, \quad \lim _{y \rightarrow 1} \frac{\frac{d}{d y} p_{i}(y)}{p_{i}(y)}=\frac{1}{2} \frac{1+\lambda_{i}(h)}{1-\lambda_{i}(h)}, \\
& \lim _{y \rightarrow 1} \frac{d}{d y}\left(y \frac{\frac{d}{d y} p_{i}(y)}{p_{i}(y)}\right)=-\frac{\left(1-2 w_{i}\right) \lambda_{i}(h)}{\left(1-\lambda_{i}(h)\right)^{2}} .
\end{aligned}
$$

Proof. For (i) see the proof of [3, Proposition 5.2]. Statement (ii) follows from a similar elementary but tedious computation.

Let $I_{0}:=\{1, \ldots, n\}$ and let $H \subset G$ be a subgroup of $G$. For a subset $I \subset I_{0}(I=\emptyset$ is admitted) let $H^{I}$ be the maximal subgroup of $H$ fixing the coordinates $x_{i}, i \in I$.

Lemma 12. Let $H \subset G$ be a subgroup of $G$ and $i \in I_{0}$. Then

$$
\sum_{h \in H \backslash H^{\{i\}}} \frac{1+\lambda_{i}(h)}{1-\lambda_{i}(h)}=0
$$

Proof. One has

$$
\sum_{h \in H \backslash H^{\{i\}}} \frac{1+\lambda_{i}(h)}{1-\lambda_{i}(h)}=\sum_{h \in H \backslash H^{\{i\}}} \frac{1}{1-\lambda_{i}(h)}+\sum_{h \in H \backslash H^{\{i\}}} \frac{1}{\lambda_{i}\left(h^{-1}\right)-1}=0 .
$$


Proposition 13. Let $r \in \mathbb{Z}, r \geq 2$, and $\zeta_{r}=\mathbf{e}[1 / r]$ be a primitive rth root of unity. Then one has

$$
-\sum_{k=1}^{r-1} \frac{\zeta_{r}^{k}}{\left(1-\zeta_{r}^{k}\right)^{2}}=\frac{r^{2}-1}{12} .
$$

Proof. One has

$$
-\sum_{k=1}^{r-1} \frac{\zeta_{r}^{k}}{\left(1-\zeta_{r}^{k}\right)^{2}}=\lim _{t \rightarrow 1} q^{\prime}(t) \text { where } q(t):=-\sum_{k=1}^{r-1} \frac{1}{1-\zeta_{r}^{k} t} .
$$

One can easily see that

$$
q(t)=\frac{-r\left(\sum_{k=0}^{r-2} t^{k}\right)+\sum_{k=0}^{r-2}(k+1) t^{k}}{\sum_{k=0}^{r-1} t^{k}} .
$$

This implies

$$
\lim _{t \rightarrow 1} q^{\prime}(t)=\frac{1}{r^{2}}\left[\sum_{k=1}^{r-2} k(k-r+1) r-\left(\sum_{\ell=1}^{r-1}(\ell-r)\right)\left(\sum_{k=1}^{r-1} k\right)\right]=\frac{r^{2}-1}{12} .
$$

Corollary 14. Let $H \subset G$ be a subgroup of $G$ and $i \in I_{0}$. Then

$$
-\sum_{h \in H \backslash H^{\{i\}}} \frac{\lambda_{i}(h)}{\left(1-\lambda_{i}(h)\right)^{2}}=\frac{\left|H \cap H^{\{i\}}\right|\left(\left|H / H \cap H^{\{i\}}\right|^{2}-1\right)}{12} .
$$

Proof. The image of the factor group $H / H \cap H^{\{i\}}$ under the induced character $\lambda_{i}$ : $H / H \cap H^{\{i\}} \rightarrow \mathbb{C}^{*}$ is a finite abelian subgroup of the unit circle $S^{1}$ and hence cyclic. Therefore, the formula follows from Proposition 13.

Let

$$
((x)):= \begin{cases}x-[x]-\frac{1}{2} & \text { if } x \in \mathbb{R}, x \notin \mathbb{Z}, \\ 0 & \text { if } x \in \mathbb{Z} .\end{cases}
$$

Proposition 15. Let $r \in \mathbb{Z}, r \geq 2, \zeta_{r}=\mathbf{e}[1 / r]$ be a primitive $r$ th root of unity, and $a, b$ be integers satisfying $0<a, b<r$. Then one has

$$
\frac{1}{4 r} \sum_{\substack{k=1, r \nmid a k, b k}}^{r-1} \frac{1+\zeta_{r}^{a k}}{1-\zeta_{r}^{a k}} \frac{1+\zeta_{r}^{b k}}{1-\zeta_{r}^{b k}}=-\sum_{k=1}^{r-1}\left(\left(\frac{a k}{r}\right)\right)\left(\left(\frac{b k}{r}\right)\right) .
$$

Remark 16. The right-hand side of the formula of Proposition 15 is a generalized Dedekind sum and Proposition 15 is a slight generalization of [7, 5.2 Theorem 1], since

$$
\frac{1+\mathbf{e}[x]}{1-\mathbf{e}[x]}=\sqrt{-1} \cot \pi x
$$

for any real number $x$. The difference is that [7, 5.2 Theorem 1] is only formulated for integers $a, b$ prime to $r$. 
Proof of Proposition 15. We follow the proof of [7, 5.2 Theorem 1]. For simplicity, we assume $b=1$. By the formula [7, $5.2(2)]$, which goes back to Eisenstein [5], we have

$$
\left(\left(\frac{q}{r}\right)\right)=-\frac{1}{2 r} \sum_{\ell=1}^{r-1} \zeta_{r}^{\ell q} \frac{\zeta_{r}^{\ell}+1}{\zeta_{r}^{\ell}-1}
$$

for any integers $q$ and $r$. (Note that there is a minor misprint in [7, 5.2 (2)].) Applying this formula, we get

$$
\begin{aligned}
& \sum_{\ell=1}^{r-1}\left(\left(\frac{a \ell}{r}\right)\right)\left(\left(\frac{\ell}{r}\right)\right) \\
& =\sum_{\ell=1}^{r}\left(\left(\frac{a \ell}{r}\right)\right)\left(\left(\frac{\ell}{r}\right)\right)=\frac{1}{4 r^{2}} \sum_{\ell=1}^{r} \sum_{m=1}^{r-1} \sum_{k=1}^{r-1} \zeta_{r}^{(m+a k) \ell} \frac{\zeta_{r}^{m}+1}{\zeta_{r}^{m}-1} \frac{\zeta_{r}^{k}+1}{\zeta_{r}^{k}-1} \\
& =\frac{1}{4 r} \sum_{\substack{k=1, r \ngtr a k}}^{r-1} \frac{\zeta_{r}^{-a k}+1}{\zeta_{r}^{-a k}-1} \frac{\zeta_{r}^{k}+1}{\zeta_{r}^{k}-1}=-\frac{1}{4 r} \sum_{\substack{k=1, r \ngtr a k}}^{r-1} \frac{1+\zeta_{r}^{a k}}{1-\zeta_{r}^{a k}} \frac{1+\zeta_{r}^{k}}{1-\zeta_{r}^{k}},
\end{aligned}
$$

since

$$
\sum_{\ell=1}^{r} \zeta_{r}^{(m+a k) \ell}= \begin{cases}0 & \text { if } m+a k \not \equiv 0 \bmod r \\ r & \text { if } m+a k \equiv 0 \bmod r\end{cases}
$$

Corollary 17. Let $K \subset J \subset I_{0}$. Then

$$
\frac{1}{4} \sum_{h \in G^{K}}\left(\sum_{\substack{j \in J \backslash K \\ \lambda_{j}(h) \neq 1}} \frac{1+\lambda_{j}(h)}{1-\lambda_{j}(h)}\right)^{2}=-\left|G^{K}\right| \sum_{h \in G^{K}}\left(\sum_{j \in J \backslash K}\left(\left(a_{j} w_{j}\right)\right)\right)^{2},
$$

where $\lambda_{j}(h)=\mathbf{e}\left[a_{j} w_{j}\right]$ for all $h \in G^{K}$ and $j \in J \backslash K$.

Proof. This follows from Proposition 15 by the same arguments as in the proof of Corollary 14.

Proposition 18. For a non-degenerate weighted homogeneous polynomial $f$, one has

$$
\mu_{(f, G)}=\frac{(-1)^{n}}{|G|}\left\{\sum_{I \subset I_{0}} \prod_{i \in I}\left(1-\frac{1}{w_{i}}\right)\left[\sum_{I \subset J \subset I_{0}}(-1)^{|J|-|I|}\left|G^{J}\right|^{2}\right]\right\} .
$$

Proof. Let $J \subset I_{0}$. Let $G_{J}$ be the set of elements $g \in G$ with $\lambda_{j}(g)=1$ for $j \in J$ and $\lambda_{j}(g) \neq 1$ for $j \notin J$, i.e., the set of elements of $G$ which fix the coordinates $x_{j}, j \in J$, and only these coordinates. Then

$$
\left|G_{J}\right|=\sum_{\substack{K, J \subset K \subset I_{0}}}(-1)^{|K|-|J|}\left|G^{K}\right| .
$$

Let $I \subset J$. Let $G_{I, J}$ be the set of elements $g$ of $G$ with $\lambda_{i}(g)=1$ for $i \in I$ and $\Lambda_{j}(g) \neq 1$ for $j \in J \backslash I$ (and $\lambda_{k}(g)$ arbitrary for $k \in I_{0} \backslash J$ ). Then

$$
\left|G_{I, J}\right|=\sum_{\substack{K, K \\ I \subset K \subset J}}(-1)^{|K|-|I|}\left|G^{K}\right|
$$


By Proposition 11 one has

$$
\begin{aligned}
\lim _{y \rightarrow 1} \chi(f, G)(y) & =\frac{(-1)^{n}}{|G|} \sum_{\substack{J, J \subset I_{0}}}\left|G_{J}\right|\left(\sum_{\substack{I, j \\
I \subset J}} \prod_{i \in I}\left(1-\frac{1}{w_{i}}\right)\left|G_{I, J}\right|\right) \\
& =\frac{(-1)^{n}}{|G|} \sum_{\substack{I, I \\
I \subset I_{0}}} \prod_{i \in I}\left(1-\frac{1}{w_{i}}\right)\left(\sum_{\substack{J, j \\
I \subset J \subset I_{0}}}\left|G_{J}\right|\left|G_{I, J}\right|\right) .
\end{aligned}
$$

Now let $I \subset I_{0}$ be fixed. Then

$$
\begin{aligned}
\sum_{\substack{J, I \subset J \subset I_{0}}}\left|G_{J}\right|\left|G_{I, J}\right| & =\sum_{\substack{J, I \subset J \subset I_{0}}}\left(\sum_{\substack{K, J \subset K \subset I_{0}}}(-1)^{|K|-|J|}\left|G^{K}\right|\right)\left(\sum_{\substack{L, I \subset L \subset J}}(-1)^{|L|-|I|}\left|G^{L}\right|\right) \\
& =\sum_{\substack{L, I \subset L \subset I_{0}}} \sum_{\substack{L \subset K, L \subset K \subset I_{0}}}\left(\sum_{\substack{J, L \subset J \subset K}}(-1)^{|K|+|L|-|I|-|J|}\right)\left|G^{K}\right|\left|G^{L}\right| \\
& =\sum_{\substack{K, I \subset K \subset I_{0}}}(-1)^{|K|-|I|}\left|G^{K}\right|^{2},
\end{aligned}
$$

since for fixed $L \subset I_{0}$ and $K \subset I_{0}$ with $L \subset K$

$$
\sum_{\substack{J J \\ L \subset J \subset K}}(-1)^{|K|+|L|-|I|-|J|}=(-1)^{|K|-|I|}(1-1)^{|K|-|L|}= \begin{cases}(-1)^{|K|-|I|} & \text { for } L=K, \\ 0 & \text { otherwise. }\end{cases}
$$

Now, we are ready to state the main result of our paper.

Theorem 19. For a non-degenerate weighted homogeneous polynomial $f$, one has

$$
\operatorname{Var}_{(f, G)}=\sum_{p, q \in \mathbb{Q}}(-1)^{(p-n)+q}\left(q-\frac{n}{2}\right)^{2} h^{p, q}(f, G)=\frac{1}{12} \hat{c} \cdot \mu_{(f, G)} .
$$

Proof. We use the notation introduced in the proof of Proposition 18. By Proposition 11 and Lemma 12 we have

$$
\lim _{y \rightarrow 1} \frac{d}{d y}\left(y \frac{d}{d y} \chi(f, G)(y)\right)=A+B+C,
$$

where

$$
A:=\frac{(-1)^{n}}{|G|} \sum_{\substack{J, J \subset I_{0}}} \sum_{g \in G_{J}}\left(\operatorname{age}(g)-\frac{n-n_{g}}{2}\right)^{2}\left[\sum_{\substack{I, I \subset J}} \prod_{i \in I}\left(1-\frac{1}{w_{i}}\right)\left|G_{I, J}\right|\right],
$$




$$
\begin{aligned}
B:= & \frac{(-1)^{n}}{|G|} \sum_{\substack{J, J \subset I_{0}}}\left|G_{J}\right|\left[\sum_{\substack{I, I \subset J}} \prod_{i \in I}\left(1-\frac{1}{w_{i}}\right) \sum_{h \in G_{I, J}} \frac{1}{4}\left(\sum_{j \in J \backslash I} \frac{1+\lambda_{j}(h)}{1-\lambda_{j}(h)}\right)^{2}\right], \\
C:= & \frac{(-1)^{n}}{|G|} \sum_{\substack{J, J \subset I_{0}}}\left|G_{J}\right| \\
& \times\left[\sum_{\substack{I, I \subset J}} \prod_{i \in I}\left(1-\frac{1}{w_{i}}\right)\left(\left|G_{I, J}\right|\left(\sum_{i \in I} \frac{1-2 w_{i}}{12}\right)-\sum_{h \in G_{I, J}} \sum_{\substack{j \in J, j \notin I}} \frac{\left(1-2 w_{j}\right) \lambda_{j}(h)}{\left(1-\lambda_{j}(h)\right)^{2}}\right)\right] .
\end{aligned}
$$

(a) We first show that $A+B=0$. We first take the sums in $A$ and $B$ in a different order:

$$
\begin{aligned}
A & =\frac{(-1)^{n}}{|G|} \sum_{\substack{I, I \subset I_{0}}} \prod_{i \in I}\left(1-\frac{1}{w_{i}}\right) A_{I}, A_{I}:=\sum_{\substack{J, I \subset J \subset I_{0}}} \sum_{g \in G_{J}}\left(\operatorname{age}(g)-\frac{n-n_{g}}{2}\right)^{2}\left|G_{I, J}\right|, \\
B & =\frac{(-1)^{n}}{|G|} \sum_{\substack{I,, I \subset I_{0}}} \prod_{i \in I}\left(1-\frac{1}{w_{i}}\right) B_{I}, \\
B_{I} & :=\sum_{\substack{J, I \subset J \subset I_{0}}}\left|G_{J}\right|\left(\sum_{h \in G_{I, J}} \frac{1}{4}\left(\sum_{j \in J \backslash I} \frac{1+\lambda_{j}(h)}{1-\lambda_{j}(h)}\right)^{2}\right) .
\end{aligned}
$$

Now let $I \subset I_{0}$ be fixed. Let $\lambda_{i}(g)=\mathbf{e}\left[a_{i} w_{i}\right]$. Then, we have on the one hand:

$$
\begin{aligned}
A_{I} & =\sum_{\substack{J, I \subset J \subset I_{0}}}\left|G_{I, J}\right| \sum_{g \in G_{J}}\left(\sum_{j \in I_{0} \backslash J}\left(\left(a_{j} w_{j}\right)\right)\right)^{2} \\
& =\sum_{\substack{J, I \subset J \subset I_{0}}}\left|G_{I, J}\right| \sum_{\substack{K, J \subset K \subset I_{0}}}(-1)^{|K|-|J|} \sum_{g \in G^{K}}\left(\sum_{j \in I_{0} \backslash K}\left(\left(a_{j} w_{j}\right)\right)\right)^{2} .
\end{aligned}
$$

On the other hand, we have by Corollary 17

$$
\begin{aligned}
B_{I} & =\sum_{\substack{J, J \\
I \subset J \subset I_{0}}}\left|G_{J}\right| \sum_{h \in G_{I, J}} \frac{1}{4}\left(\sum_{j \in J \backslash I} \frac{1+\lambda_{j}(h)}{1-\lambda_{j}(h)}\right)^{2} \\
& =\sum_{\substack{J, J \\
I \subset J \subset I_{0}}}\left|G_{J}\right| \sum_{\substack{K,, I \subset K \subset J}}(-1)^{|K|-|I|} \sum_{h \in G^{K}} \frac{1}{4}\left(\sum_{j \in J \backslash K} \frac{1+\lambda_{j}(h)}{1-\lambda_{j}(h)}\right)^{2} \\
& =-\sum_{\substack{J, I \subset J \subset I_{0}}}\left|G_{J}\right| \sum_{\substack{K, I \subset K \subset J}}(-1)^{|K|-|I|}\left|G^{K}\right| \sum_{h \in G^{K}}\left(\sum_{j \in J \backslash K}\left(\left(a_{j} w_{j}\right)\right)\right)^{2} .
\end{aligned}
$$


For $I \subset K \subset J \subset I_{0}$ let

$$
s(K, J):=\sum_{g \in G^{K}}\left(\sum_{j \in J \backslash K}\left(\left(a_{j} w_{j}\right)\right)\right)^{2} .
$$

Then

$$
\begin{aligned}
A_{I} & =\sum_{\substack{K, I \subset K \subset I_{0}}} \sum_{\substack{J, I \subset J \subset K}}(-1)^{|K|-|J|}\left|G_{I, J}\right| s\left(K, I_{0}\right) \\
& =\sum_{\substack{K, I \subset K \subset I_{0}}} \sum_{\substack{J, I \subset J \subset K}}(-1)^{|K|-|J|}\left(\sum_{\substack{L, I \subset L \subset J}}(-1)^{|L|-|I|}\left|G^{L}\right|\right) s\left(K, I_{0}\right) \\
& =\sum_{\substack{L, I \subset L \subset I_{0}}} \sum_{\substack{K, L \subset K \subset I_{0}}}\left(\sum_{\substack{J, L \subset J \subset K}}(-1)^{|K|+|L|-|I|-|J|}\right)\left|G^{L}\right| s\left(K, I_{0}\right) \\
& =\sum_{\substack{K, I \subset K \subset I_{0}}}(-1)^{|K|-|I|}\left|G^{K}\right| s\left(K, I_{0}\right)
\end{aligned}
$$

by Formula (2.2). On the other hand, we have

$$
\begin{aligned}
B_{I} & =-\sum_{\substack{K, I \subset K \subset I_{0}}} \sum_{\substack{J, K \subset J \subset I_{0}}}(-1)^{|K|-|I|}\left|G_{J}\right|\left|G^{K}\right| s(K, J) \\
& =-\sum_{\substack{K, I \subset K \subset I_{0}}} \sum_{\substack{J, K \subset J \subset I_{0}}}(-1)^{|K|-|I|}\left(\sum_{\substack{L, J \subset L \subset I_{0}}}(-1)^{|L|-|J|}\left|G^{L}\right|\right)\left|G^{K}\right| s(K, J) \\
& =-\sum_{\substack{L, I \subset L \subset I_{0}}} \sum_{\substack{K, K, I \subset K \subset L}}\left(\sum_{\substack{J, L \subset J \subset I_{0}}}(-1)^{|K|+|L|-|I|-|J|}\right)\left|G^{L}\right|\left|G^{K}\right| s(K, J) \\
& =-\sum_{\substack{K,, I \subset K \subset I_{0}}}^{(-1)^{|K|-|I|}\left|G^{K}\right| s\left(K, I_{0}\right)=-A_{I},}
\end{aligned}
$$

again by Formula (2.2) and since $\left|G^{I_{0}}\right|=1$. This shows that $A+B=0$.

(b) We now consider the term $C$. Let $J \subset I_{0}, I \subset J$ and $j \in J, j \notin I$. Then it follows from Corollary 14 that

$$
-\sum_{h \in G_{I, J}} \frac{\lambda_{j}(h)}{\left(1-\lambda_{j}(h)\right)^{2}}=\frac{1}{12} m_{I, j}^{J},
$$

where

$$
m_{I, j}^{J}:=\sum_{\substack{K, j \notin K, I \subset K \subset J}}(-1)^{|K|-|I|}\left|G^{K \cup\{i\}}\right|\left(\left|G^{K} / G^{K \cup\{i\}}\right|^{2}-1\right) .
$$


By (a) we have

$$
\begin{aligned}
\lim _{y \rightarrow 1} \frac{d}{d y}\left(y \frac{d}{d y} \chi(f, G)(y)\right) \\
=C=\frac{(-1)^{n}}{|G|} \sum_{\substack{J, J \subset I_{0}}}\left|G_{J}\right| \\
\times\left[\sum_{\substack{I, j \\
I \subset J}} \prod_{i \in I}\left(1-\frac{1}{w_{i}}\right)\left(\left|G_{I, J}\right|\left(\sum_{i \in I} \frac{1-2 w_{i}}{12}\right)+\sum_{\substack{j \in J, j \notin I}} m_{I, j}^{J}\left(\frac{1-2 w_{j}}{12}\right)\right)\right] \\
\left.=\frac{(-1)^{n}}{|G|} \sum_{\substack{I, I \subset I_{0}}} \prod_{i \in I}\left(1-\frac{1}{w_{i}}\right)\right] \\
\times\left[\sum_{\substack{J, I \subset J \subset I_{0}}}\left|G_{J}\right|\left(\left|G_{I, J}\right|\left(\sum_{i \in I} \frac{1-2 w_{i}}{12}\right)+\sum_{\substack{j \in J, j \notin I}} m_{I, j}^{J}\left(\frac{1-2 w_{j}}{12}\right)\right)\right]
\end{aligned}
$$

Now let $I \subset I_{0}$ and $j \notin I$ be fixed. Then

$$
\begin{aligned}
\left.\sum_{\substack{J, j \in J, I \subset J \subset I_{0}}} \mid G_{J}\right] m_{I, j}^{J}= & \sum_{\substack{J, j \in J, I \subset J \subset I_{0}}}\left(\sum_{\substack{K, J \subset K \subset I_{0}}}(-1)^{|K|-|J|}\left|G^{K}\right|\right) \\
& \times\left(\sum_{\substack{L, j \notin L, I \subset L \subset J}}(-1)^{|L|-|I|}\left|G^{L \cup\{j\}}\right|\left(\left|G^{L} / G^{L \cup\{j\}}\right|^{2}-1\right)\right) \\
= & \sum_{\substack{L, j \notin L, I \subset L \subset I_{0}}} \sum_{\substack{K, j \in K \\
L \subset K \subset I_{0}}}\left(\sum_{\substack{J, j \in J, L \subset J \subset K}}(-1)^{|K|+|L|-|I|-|J|}\right) \\
& \times\left|G^{K}\right|\left|G^{L \cup\{j\}}\right|\left(\left|G^{L} / G^{L \cup\{j\}}\right|^{2}-1\right) .
\end{aligned}
$$

Since $j \notin L$ but $j \in J$, the case $J=L$ and hence also $K=L$ is excluded in the sum

$$
\sum_{\substack{J, j \in J, L \subset J \subset K}}(-1)^{|K|+|L|-|I|-|J|}
$$

Therefore

$$
\sum_{\substack{J, j \in J, L \subset J \subset K}}(-1)^{|K|+|L|-|I|-|J|}= \begin{cases}(-1)^{|L|-|I|} & \text { for } K=L \cup\{j\} \\ 0 & \text { otherwise. }\end{cases}
$$


Hence, we obtain

$$
\begin{aligned}
\left.\sum_{\substack{J, j \in J, I \subset J \subset I_{0}}} \mid G_{J}\right] m_{I, j}^{J} & =\sum_{\substack{L, j \notin L, I \subset L \subset I_{0}}}(-1)^{|L|-|I|}\left|G^{L \cup\{j\}}\right|^{2}\left(\left|G^{L} / G^{L \cup\{j\}}\right|^{2}-1\right) . \\
& =\sum_{\substack{L, j \notin L, I \subset L \subset I_{0}}}(-1)^{|L|-|I|}\left(\left|G^{L}\right|^{2}-\left|G^{L \cup\{j\}}\right|^{2}\right) \\
& =\sum_{\substack{K, I \subset K \subset I_{0}}}(-1)^{|K|-|I|}\left|G^{K}\right|^{2} .
\end{aligned}
$$

Therefore, the statement follows from Proposition 18.

\section{Variance of the exponents for cusp singularities with group actions}

Let $f\left(x_{1}, x_{2}, x_{3}\right):=x_{1}^{\alpha_{1}}+x_{2}^{\alpha_{2}}+x_{3}^{\alpha_{3}}-x_{1} x_{2} x_{3}$ and $G$ be a finite subgroup of $S L_{n}(\mathbb{C})$ acting diagonally on $\mathbb{C}^{n}$ under which $f$ is invariant. Let $K_{i} \subset G$ be the maximal subgroup fixing the coordinate $x_{i}, i=1,2,3$. Define numbers $\gamma_{1}, \ldots, \gamma_{s}$ by

$$
\left(\gamma_{1}, \ldots, \gamma_{s}\right)=\left(\frac{\alpha_{i}}{\left|G / K_{i}\right|} *\left|K_{i}\right|, i=1,2,3\right),
$$

where we omit numbers which are equal to one on the right-hand side. Define a number $\chi_{(f, G)}$ by

$$
\chi_{(f, G)}:=2-2 j_{G}+\sum_{i=1}^{s}\left(\frac{1}{\gamma_{i}}-1\right) .
$$

Lemma 20. Let the pair $(f, G)$ be as above.

(i) The Milnor number of the pair $(f, G)$ is given by

$$
\mu_{(f, G)}=2-2 j_{G}+\sum_{i=1}^{s}\left(\gamma_{i}-1\right) .
$$

(ii) The set of exponents for the pair $(f, G)$ is given by

$$
\begin{gathered}
\{1,2\} \coprod\left\{\frac{1}{\gamma_{1}}+1, \frac{2}{\gamma_{1}}+1, \ldots, \frac{\gamma_{1}-1}{\gamma_{1}}+1\right\} \\
\coprod\left\{\frac{1}{\gamma_{2}}+1, \frac{2}{\gamma_{2}}+1, \ldots, \frac{\gamma_{2}-1}{\gamma_{2}}+1\right\} \coprod \ldots \\
\cdots \coprod\left\{\frac{1}{\gamma_{s}}+1, \frac{2}{\gamma_{s}}+1, \ldots, \frac{\gamma_{s}-1}{\gamma_{s}}+1\right\}
\end{gathered}
$$

Proof. See Corollary 5.13 and the proof of Theorem 5.12 of [4].

We have the following formula for the variance. Note that we have $\hat{c}=1$ by Theorem 4.

Theorem 21. Let the pair $(f, G)$ be as above. The variance of the set of exponents of $(f, G)$ is given by

$$
\operatorname{Var}_{(f, G)}=\frac{1}{12} \mu_{(f, G)}+\frac{1}{6} \chi_{(f, G)}=\frac{1}{12} \hat{c} \cdot \mu_{(f, G)}+\frac{1}{6} \chi_{(f, G)} .
$$


Proof. Some elementary calculation yields the statement.

Note that the pair $(f, G)$ can be considered as a mirror partner of the orbifold curve (Deligne-Mumford stack) $\mathcal{C}$ which is a smooth projective curve of genus $j_{G}$ with $s$ isotropic points of orders $\gamma_{1}, \ldots, \gamma_{s}$ (cf. Theorem 7.1 of [4]). The above formula for the variance is compatible with this observation. In particular, the dimension of $\mathcal{C}$ is $1, \mu_{(f, G)}$ is the orbifold Euler number $\chi(\mathcal{C})$ of $\mathcal{C}$ and $\chi_{(f, G)}$ is the orbifold Euler characteristic of $\mathcal{C}$, which is the degree of the first Chern class $c_{1}(\mathcal{C})$ of $\mathcal{C}$. Applying this to the formula in Theorem 1, we recover the equation (3.3).

\section{Acknowledgments}

This work was supported by the DFG-program SPP1388 "Representation Theory" (Eb 102/6-1). The second named author is also supported by JSPS KAKENHI Grant Number 24684005 . We are very grateful to the anonymous referee for carefully reading our paper, drawing our attention to a serious gap in the proof of the main result in the first version, and for most valuable comments which led to a major revision of the article.

\section{References}

[1] V.I. Arnold, S.M. Gusein-Zade and A.N. Varchenko, Singularities of differentiable maps, Volume II, Birkhäuser, Boston Basel Berlin 1988.

[2] L. Borisov, On Betti numbers and Chern classes of varieties with trivial odd cohomology groups, arXiv: alg-geom/9703023.

[3] A. Dimca, Monodromy and Hodge theory of regular functions. In 'New developments in singularity theory' (Cambridge, 2000), NATO Science Ser. II Math. Phys. Chem., 21, Kluwer Academic Publ., Dordrecht, 2001, 257-278.

[4] W. Ebeling and A. Takahashi, Mirror symmetry between orbifold curves and cusp singularities with group action, Int. Math. Res. Not. 2013(10) (2013), 2240-2270.

[5] G. Eisenstein, Théorèmes arithmétiques, J. Reine Angew. Math. 27 (1844), 281-283.

[6] C. Hertling, Frobenius manifolds and variance of the spectral numbers. In 'New developments in singularity theory' (Cambridge, 2000), NATO Science Ser. II Math. Phys. Chem., 21, Kluwer Academic Publ., Dordrecht, 2001, 235-255.

[7] F. Hirzebruch and D. Zagier, The Atiyah-Singer theorem and elementary number theory, Publish or Perish, Inc., Berkeley, 1974.

[8] Y. Ito and M. Reid, The McKay correspondence for finite subgroups of SL(3, C). In 'Higherdimensional complex varieties' (Trento, 1994), de Gruyter, Berlin, 1996, 221-240.

[9] A. Libgober and J. Wood, Uniqueness of the complex structure on Kähler manifolds of certain homotopy types, J. Differ. Geom. 32(1) (1990), 139-154.

[10] J.H.M. Steenbrink, Mixed Hodge structure on the vanishing cohomology. In 'Real and Complex Singularities', Proc. Nordic Summer School, Oslo, 1976, 525-563.

Institut für Algebraische Geometrie, Leibniz Universität Hannover, Postfach 6009, D-30060 HANNOVER, GERMANY

E-mail address: ebeling@math.uni-hannover.de

Department of Mathematics, Graduate School of Science, Osaka University, Toyonaka OSAKA, 560-0043, JAPAN

E-mail address: takahashi@math.sci.osaka-u.ac.jp 
